\title{
DESEMPENHO FUNCIONAL NAS ATIVIDADES BÁSICAS DE VIDA DIÁRIA EM CRIANÇAS COM DEFICIÊNCIA VISUAL
}

DOI: $10.22289 / 2446-922 X . V 7 N 2 A 8$

\author{
Marcela Aparecida dos Santos ${ }^{1}$ \\ Marcia Caires Bestilleiro Lopes \\ Célia Regina Nakanami
}

\section{RESUMO}

A visão é um dos mais importantes sentidos no desenvolvimento normal da criança. Quando há o comprometimento dessa via sensorial, a criança pode apresentar dificuldades no desenvolvimento neuropsicomotor, na relação familiar e ainda nas habilidades da vida diária. Avaliar o desempenho das habilidades funcionais e de assistência dos pais/cuidadores nas atividades básicas de vida diária em crianças com deficiência visual em comparação ao desempenho de crianças sem deficiência visual. Este estudo prospectivo, observacional e transversal foi realizado no Ambulatório de Estimulação Visual Precoce - Setor de Baixa Visão e Reabilitação Visual do Instituto da Visão do Departamento de Oftalmologia da Universidade Federal de São Paulo. O instrumento utilizado foi o PEDI - Inventário de Avaliação Pediátrica de Incapacidade, aplicado através de entrevista individual com o responsável pela criança nas áreas de Habilidades Funcionais e Nível de Assistência do Cuidador. O PEDI é subdividido em itens: autocuidado, mobilidade e função social. Segundo os dados obtidos, as crianças deficientes visuais apresentaram um déficit de desempenho na área de autocuidado e são mais dependentes do cuidador na mobilidade e função social. As crianças sem deficiência visual apresentaram um menor desempenho na área de autocuidado e mesmo nível de dependência do cuidador nas três áreas. Crianças com deficiência visual necessitam de mais ajuda para desempenhar suas atividades diárias em todas as áreas funcionais se comparadas com as crianças sem deficiência visual.

Palavras-chave: Atividades Cotidianas; Terapia Ocupacional; Crianças; Deficiência Visual.

\section{FUNCTIONAL PERFORMANCE IN BASIC ACTIVITIES OF DAILY LIVING IN CHILDREN WITH VISUAL IMPAIRMENT}

\section{ABSTRACT}

The vision is one of the most importante sensesl in the normal developmental of the children. When this sensory pathway is compromised, the children may difficulties in neuropsychomotor developmental, in family relationships and even in daily life skills. To evaluate the performance of functional skills and care of parents/caregivers in basic activities of daily living in blind

\footnotetext{
${ }^{1}$ Endereço eletrônico de contato: marcelasantos.to@gmail.com

Recebido em 11/06/2021. Aprovado pelo conselho editorial para publicação em 30/07/2021.
} 
children and compare this performance with the diagnosis of children without visual impairments. This prospective, observational and cross study was carried out from June to December 2010 at the Clinic of Early Visual Stimulation-Department of Low Vision and Visual Rehabilition of the Department of Ophthalmology, Federal University of São Paulo. The PEDIInventory Pediatric Evaluation of Disability tool was used and an interview with the caretaker was applied in the areas of Functional Skills and Caregiver Assistance Level. The PEDI was subdivided into three specific areas: self-care, mobility and social function areas. According to data obtained, visually impaired children showed a performance deficit in the specific self-care area and are more dependent of the caregiver on mobility and social role. Children without visual impairment showed a lower performance in the self-care area and the same level of dependence of the caregiver in the three areas. Visually impaired children need more help to perform their daily activities in all functional areas compared to children without visual impairment.

Keywords: Activities of Daily Living; Occupation Therapy; Children; Visual Impairment.

\section{RENDIMIENTO FUNCIONAL EM LAS ACTIVIDADES BÁSICAS DE LA VIDA DIARIA EN NIÑOS COM DISCAPACIDAD VISUAL}

\section{RESUMEN}

La vison es uno de los sentidos más importantes en el desarrollo normal de un niño. Cuando esta vía sensorial se ve comprometida, el niño puede tener dificultades en el desarrrollo neuropsicomotor, en las relaciones familiares e incluso en las habilidades de la vida diaria. Evaluar el desempeño de las habilidades funcionales y asistenciales de los padres/cuidadores en las actividades básicas de la vida diaria en niños con discapacidad visual en comparación con el desempeño de niños sin discapacidad visual. Este studio prospective, observacional y transversal se realize en el Ambulatorio de Estimulación Visual Temprana-Sector Baja Visión y Rehabilitación Visual del Instituto de Visión del Departamento de Oftalmologia d ela Universidad Federal de São Paulo. El instrument utilizado fue el PEDI- Inventario de Evalución de Discapacidad Pediátrica, aplicado a través de entrevistas individuales con el responsible del niño en las áreas de Habilidades Funcionales y Nivel de Asistencia al Cuidador.PEDI se subdivide en ítems: autocuidado, movilidad y función social. Según los datos obtenidos, los niños con discapacidad visual mostraron un déficit de desempeño en el área del autocuidado y son más dependientes del cuidador en términos de movilidad y función social. Los niños sin discapacidad visual mostraron un menor desempeño en el área de autocuidado y el mismo nivel de dependencia del cuidador en las tres áreas. Los niños con discapacidad visual necesitan más ayuda para realizar sus actividades diarias en todas las áreas funcionales en comparación con los niños sin discapacidad visual.

Palabras clave: Actividades Cotidianas; Terapia Ocupacional; Niños; Discapacidad Visual. 


\section{INTRODUÇÃO}

Atualmente muitas crianças vêm sendo diagnosticadas com baixa visão congênita ou adquirida, ou seja, possuem visão residual, que pode ser utilizada e melhorada por meio de procedimentos terapêuticos da habilitação e reabilitação visual e recursos especiais.

As principais causas de deficiência visual em crianças nos países desenvolvidos são consideradas as doenças genéticas, congênitas ou perinatais (Verzoni, Zin, \& Barbosa, 2017), e no Brasil a deficiência visual é decorrente principalmente de rubéola, toxoplasmose, catarata congênita, glaucoma congênito, doenças hereditárias da retina e prematuridade (Haddad, Correa, Wilson, \& Newton, 2006).

A deficiência visual pode resultar em um distúrbio ocular ou distúrbio cortical, classificando o indivíduo em cego (amaurótico), quando o prejuízo visual se encontra em níveis incapacitantes para realizar tarefas do cotidiano; ou indivíduo com baixa visão (visão subnormal), que mesmo após o tratamento e/ou correção refrativa, possuem acuidade visual comprometida e percepção de luz ou campo visual inferior a $10^{\circ}$ de campo central, sendo capaz de realizar algumas tarefas fazendo uso da visão residual (Ottaiano, Ávila, \& Umbelino, 2019).

A baixa visão está muito relacionada com a qualidade da acuidade visual (acuidade de resolução de grades) da criança, sendo esta uma função visual importante para o desenvolvimento psicomotor e visual, assim como no reconhecimento de objetos e detalhes (Kwon, Legge, Fang, Cheong, \& He, 2009; Lopes, 2014). Alguns autores descrevem que a acuidade de resolução de grades de um bebê de 01 mês corresponde a 20/600, chegando aos valores próximo de um adulto entre 03 e 05 anos de idade (Mayer, Beiser, Warner, Pratt, Raye, \& Lang, 1995; Salomão \& Ventura, 1995; Costa, Oliveira, Bergamasco \& Ventura, 2006).

A visão é um dos mais importantes sentidos no desenvolvimento normal da criança (Chak \& Rahi, 2007). Quando há o comprometimento dessa via sensorial, a criança pode apresentar dificuldades no desenvolvimento neuropsicomotor, no desempenho de algumas habilidades específicas, na relação familiar e participação em seu meio, e ainda nas habilidades da vida diária (Graziano \& Leone, 2005; Boulton, Haines, Smyth, \& Fielder, 2006; Brandão, Vasconcelos, Tibúrcio, Rossi, \& Andrade, 2019).

A visão também é fundamental para a criança, pois fornece informações ambientais que nenhum outro órgão sensorial é capaz de proporcionar (Mancini, Braga, Albuquerque, Ramos, \& Chagas, 2010). Os estímulos visuais recebidos do ambiente provocam modificações na maturação neurológica da visão da criança. Essas modificações podem, por sua vez, alterar a 
habilidade em assimilar os estímulos que recebe do ambiente (Lipsitt, 1986). Cabe ressaltar que a visão é um dos sentidos mais importantes, fornece dados do meio, organiza outras informações sensoriais; e o desenvolvimento das capacidades visuais ao longo dos primeiros meses de vida é uma ação coordenada que envolve aspectos sensoriais e motores (Scholl, 1986; Daw, 1995).

A baixa visão pode limitar experiências de vida, como a velocidade na realização das tarefas, o desenvolvimento motor, as habilidades cognitivas e de aprendizagem e 0 desenvolvimento socioemocional, impactando na funcionalidade e independência da criança e comprometendo sua qualidade de vida, principalmente quando a criança não é estimulada (Gieser, 2004; Silva \& Airoldi, 2014).

Uma das áreas que compromete o desenvolvimento da criança com deficiência visual em diversas fases do seu crescimento, relaciona-se ao desempenho nas atividades da vida diária que, para a Associação Americana de Terapia Ocupacional (AOTA, 2008), refere-se às atividades básicas do dia-a-dia importantes para viver em sociedade, permitir sobrevivência e bem-estar. E estas atividades de vida diária compreendem atividades fundamentais para a sobrevivência, como comer, manter-se limpo, participar de atividades sociais, realizar serviços domésticos, dentre outros (Finger, 1986).

As crianças com deficiência visual podem encontrar dificuldades para realizar estas atividades e tarefas de rotina diária. Sendo assim, faz-se necessário verificarmos o impacto da deficiência visual no repertório funcional destas crianças para elaboração de estratégias e conduta terapêuticas (Mancini, Braga, Albuquerque, Ramos, \& Chagas, 2010).

Além disso, na infância, em particular, as atividades de vida diária são vistas como fundamentais para o desenvolvimento biopsicossocial, pois possibilitam as primeiras formas de exploração do ambiente e o contato próximo da criança, seja ela deficiente visual ou não, com o seu cuidador principal (Vasconcelos \& Cavalcante, 2013).

À medida que a criança vai se desenvolvendo motor, cognitivo e psicologicamente, a sua interação com o ambiente torna-se mais complexa e dinâmica e sua relação com as pessoas, objetos e símbolos, mais consistentes. Esse desenvolvimento possibilita à criança criar, aos poucos, suas próprias rotinas na vida diária, como as relacionadas aos cuidados pessoais, comunicação, mobilidade, entre outras ocupações (AOTA, 2008; Bronfenbrenner, 2011).

O PEDI-Inventário de Avaliação Pediátrica de Incapacidade (PEDI) foi desenvolvido com o intuito de fornecer uma descrição mais detalhada do desempenho funcional da criança, predizer seu desempenho futuro e documentar mudanças longitudinais no seu desempenho Rev. Psicol Saúde e Debate. Jul., 2021:7(2): 113-130. 
funcional (Mancini, 2005). Ele constitui uma avaliação padronizada do desempenho das crianças nas suas atividades de vida diária e nas tarefas de rotina doméstica, bem como da dependência de seus cuidadores para realização das mesmas. Este instrumento destaca-se como o mais utilizado nas pesquisas para avaliar o desempenho nessas atividades envolvendo crianças com desenvolvimento típico ou atípico (Vasconcelos \& Cavalcante, 2013).O presente estudo tem como principal objetivo avaliar o desempenho nas habilidades funcionais e a dependência da assistência dos pais/cuidadores nas atividades básicas de vida diária em crianças com deficiência visual e comparar este desempenho com crianças sem deficiência visual.

\section{MATERIAIS E MÉTODOS}

\subsection{Aspectos éticos}

Este estudo prospectivo, observacional e transversal seguiu os princípios da Declaração de Helsinque e foi encaminhado à aprovação do Comitê de Ética em Pesquisa da Universidade Federal de São Paulo, com o parecer aprovado de número 0860/10.

\subsection{Participantes}

Todos os participantes, responsáveis pelas crianças do estudo, foram informados e orientados quanto aos objetivos e importância da pesquisa, leram o Termo de Consentimento Livre e Esclarecido, concordaram e assinaram, permitindo assim a utilização dos dados neste estudo, sendo este realizado no Ambulatório de Estimulação Visual Precoce - Setor de Baixa Visão e Reabilitação Visual do Instituto da Visão do Departamento de Oftalmologia da Universidade Federal de São Paulo.

Os responsáveis pelas crianças do grupo controle também foram informados e orientados quanto aos objetivos e importância da pesquisa, leram e assinaram o Termo de Consentimento Livre e Esclarecido.

Foram avaliadas 29 crianças alocadas em dois grupos: grupo de estudo, composto por crianças do ambulatório de Estimulação Visual Precoce do Setor de Baixa Visão e Reabilitação Visual do Departamento de Oftalmologia da Unifesp, com idade entre 2-6 anos, de ambos os sexos, com o diagnóstico de Baixa Visão e 
tratamento e acompanhamento prévio realizado; grupo controle, composto por crianças voluntárias, selecionadas de forma aleatória, também com idade entre 2-6 anos, de ambos os sexos, com visão adequada a idade cronológica e sem qualquer outra comorbidade associada.

No grupo de estudo participaram 15 crianças, com 2 - 6 anos, e média de idade de 4 anos e 3 meses, sendo oito do sexo feminino e sete do sexo masculino. As patologias de acometimento desse grupo eram: 01 com Síndrome de Peters, 01 com Retinopatia da Prematuridade, 02 crianças com Amaurose Congênita de Leber, 01 com Glaucoma Congênito, 04 com Catarata Congênita, 01 com Microftalmia e Coloboma, 01 com Hemorragia Vítrea em ambos olhos (AO), 01 com Deficiência Visual Cerebral (DVC), 01 com Palidez de papila e 02 crianças com Nistagmo Congênito. No grupo controle, participaram 14 crianças, com idade entre 2 e 6, e média de idade de 3 anos e 4 meses, quatro do sexo feminino e 10 do sexo masculino. Toda a coleta do presente estudo foi realizada pelo mesmo pesquisador, que estudou e realizou um treinamento do uso do instrumento PEDI. A coleta ocorreu em um único dia, tempo médio de 3040min, com os responsáveis pelas crianças.

\subsection{Instrumento}

O instrumento utilizado foi o PEDI - Inventário de Avaliação Pediátrica de Incapacidade: Manual da Versão Brasileira Adaptada/Marisa Cotta Mancini; [com base em] Stephen M. Haley [et al.] - Belo Horizonte: UFMG (2005.) Este instrumento documenta de forma quantitativa a capacidade funcional da criança por meio de avaliação de habilidades realizadas de forma independente durante o autocuidado, mobilidade e função social (Mancini, 2005). O teste aplicado ocorreu através de entrevista, com duração de 30 à 50 minutos, pois dependia da colaboração de cada pais/responsáveis/cuidadores da criança.

Esse instrumento, o PEDI é composto em duas partes:

A Parte I - informa sobre as Habilidades Funcionais da criança para realizar suas atividades e tarefas de seu cotidiano, nas três áreas de função: autocuidado (73 itens ou atividades funcionais), mobilidade (59 itens ou atividades funcionais) e função social (65 itens ou atividades funcionais). Para cada item da Parte I, das três escalas funcionais, é atribuído o escore 1 (um ponto), se a criança for capaz de executar a Rev. Psicol Saúde e Debate. Jul., 2021:7(2): 113-130. 
atividade funcional, ou o escore 0 (zero ponto), se a criança não for capaz de executar a atividade funcional, sozinha. O escore total obtido em cada escala é o resultado do somatório do escore 1 pontuado pela criança nas atividades incluídas em cada área da função (Mancini, 2005).

A Parte II - informa sobre Nível de Assistência do Cuidador para a criança realizar 20 tarefas funcionais nas mesmas áreas de autocuidado (8 itens), mobilidade (7 itens) e função social (5 itens). Desta forma, quanto mais ajuda a criança receber de seu cuidador para realizar tarefas funcionais, menor será sua independência nestas tarefas. O escore total obtido em cada escala é o resultado do somatório dos escores (0 à 5) pontuados pela criança nas atividades incluídas em cada área da função, sendo escore 0 (Assistência Total), se a criança necessitar de assistência total do seu cuidador; escore 1 (Assistência Máxima), se o cuidador realizar mais da metade da tarefa pela criança; escore 2 (Assistência Moderada), se a criança realizar boa parte da tarefa, porém necessita de ajuda várias vezes; escore 3 (Assistência Mínima), se a criança precisar de ajuda ocasionalmente, para iniciar ou finalizar a tarefa; escore 4 (Supervisão), se a criança necessitar do cuidador para completar a tarefa através de orientações verbais ou descrição das etapas, não há contato físico; escore 5 (Independente), se a criança não necessitar do cuidador para realizar sua tarefa funcional (Mancini, 2005).

Os dados finais do teste PEDI são obtidos através do Escore Total Bruto, que representa somatório de todos os itens pontuados em cada escala e usados para o Escore Normativo e Escore Contínuo. O Escore Normativo, que informa sobre o desempenho esperado de crianças de mesma faixa etária, com desenvolvimento normal, sendo obtido pela transformação dos escores totais brutos. Para este escore, considera-se entre 30-70 dentro do intervalo de normalidade, abaixo de 30, atraso ou desempenho significativamente inferior e acima de 70 , desempenho significativamente superior ao considerado para crianças na mesma faixa etária (Mancini, 2005). E ainda, - Escore Contínuo, que informa sobre o nível de capacidade da criança, não se levando em consideração a faixa etária da mesma, pois pode ser usado para analisar o perfil de crianças com idade cronológica superior ao limite etário compreendido pelo teste PEDI (6 meses e 7 anos e 6 meses) na presença de deficiências, que interferem no seu desempenho das atividades diárias. 
No presente estudo, os escores normativos foram considerados para verificar a relação do desempenho funcional das crianças com o nível de assistência dos cuidadores.

\section{RESULTADOS}

No grupo de estudo, crianças com deficiência visual participaram 15 crianças, com 2 6 anos, e média de idade de 4 anos e 3 meses, sendo oito do sexo feminino e sete do sexo masculino. As patologias de acometimento desse grupo eram: 01 com Síndrome de Peters, 01 com Retinopatia da Prematuridade, 02 crianças com Amaurose Congênita de Leber, 01 com Glaucoma Congênito, 04 com Catarata Congênita, 01 com Microftalmia e Coloboma, 01 com Hemorragia Vítrea em ambos olhos (AO), 01 com Deficiência Visual Cerebral (DVC), 01 com Palidez de papila e 02 crianças com Nistagmo Congênito. No grupo controle, crianças sem deficiência visual, participaram 14 crianças, com idade entre 2 e 6, e média de idade de 3 anos e 4 meses, quatro do sexo feminino e 10 do sexo masculino. Para as crianças do grupo de estudo, as atividades básicas de vida diária e a relação dependente, foram encontradas as seguintes características: $60 \%$ das crianças apresentam atraso na área funcional de autocuidado, 53,4\% na área funcional da mobilidade e 46,7\% na área funcional da função social. Ainda com relação aos dados obtidos, na assistência do cuidador, notase que $40 \%$ das crianças precisam de ajuda máxima no autocuidado, 66,7\% precisam de ajuda máxima na mobilidade e $66,7 \%$ precisam de ajuda máxima na função social. No final, 12 crianças apresentaram atraso no desempenho das atividades diárias e apenas três crianças apresentaram desempenho funcional adequado para a idade nas habilidades funcionais e ajuda mínima na assistência do cuidador (Figura 1). 


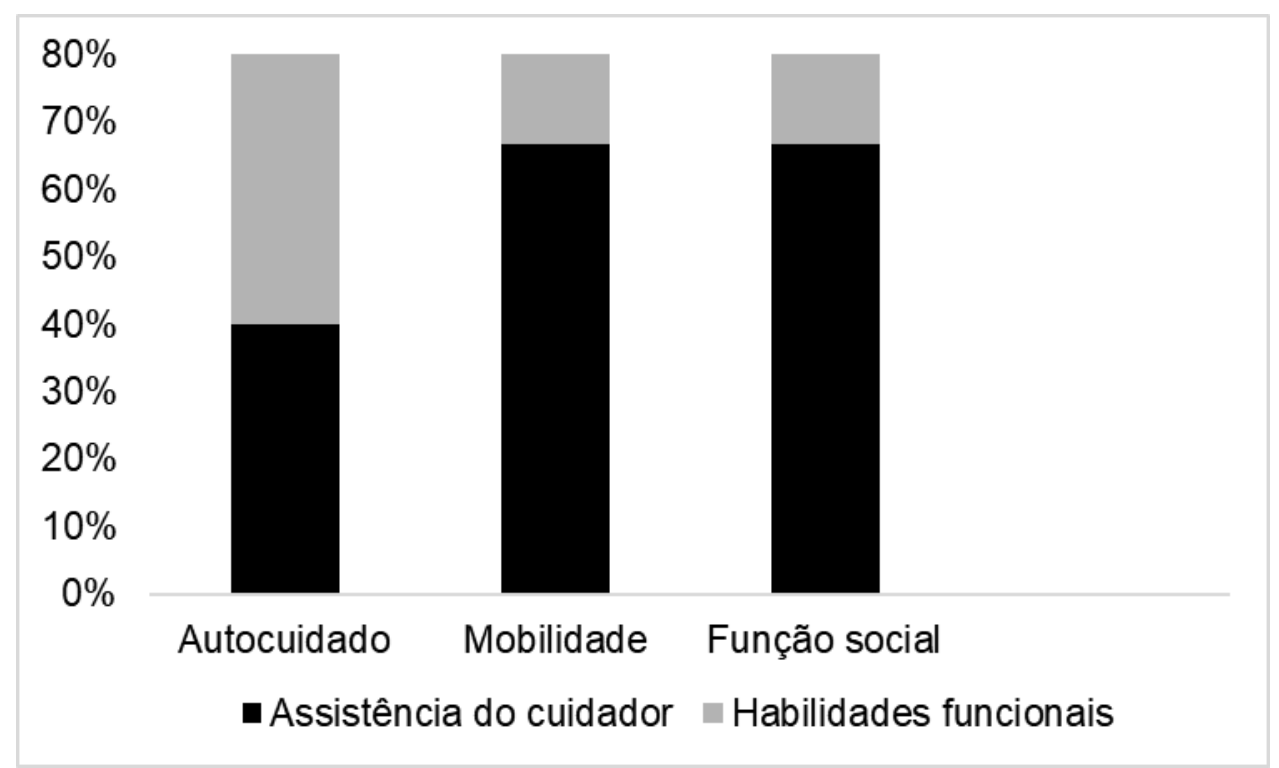

Figura 1. Descrição do desempenho nas atividades diárias e relação Habilidades Funcionais/Dependência do Cuidador nas crianças com deficiência visual.

Com relação às crianças do grupo controle, 33,4\% apresentam atraso na área funcional de autocuidado e 13,4\% na área funcional da função social, não sendo observado atraso na área funcional da mobilidade. Com relação à dependência da assistência do cuidador, 13,4\% necessitam de ajuda máxima no autocuidado, 13,4\% necessitam de ajuda máxima na mobilidade e 13,4\% de ajuda máxima na função social.

No final, um total de nove crianças apresentam desempenho funcional esperado para a idade e cinco crianças tiveram alteração funcional nas suas atividades de vida diária representada na Figura 2. 


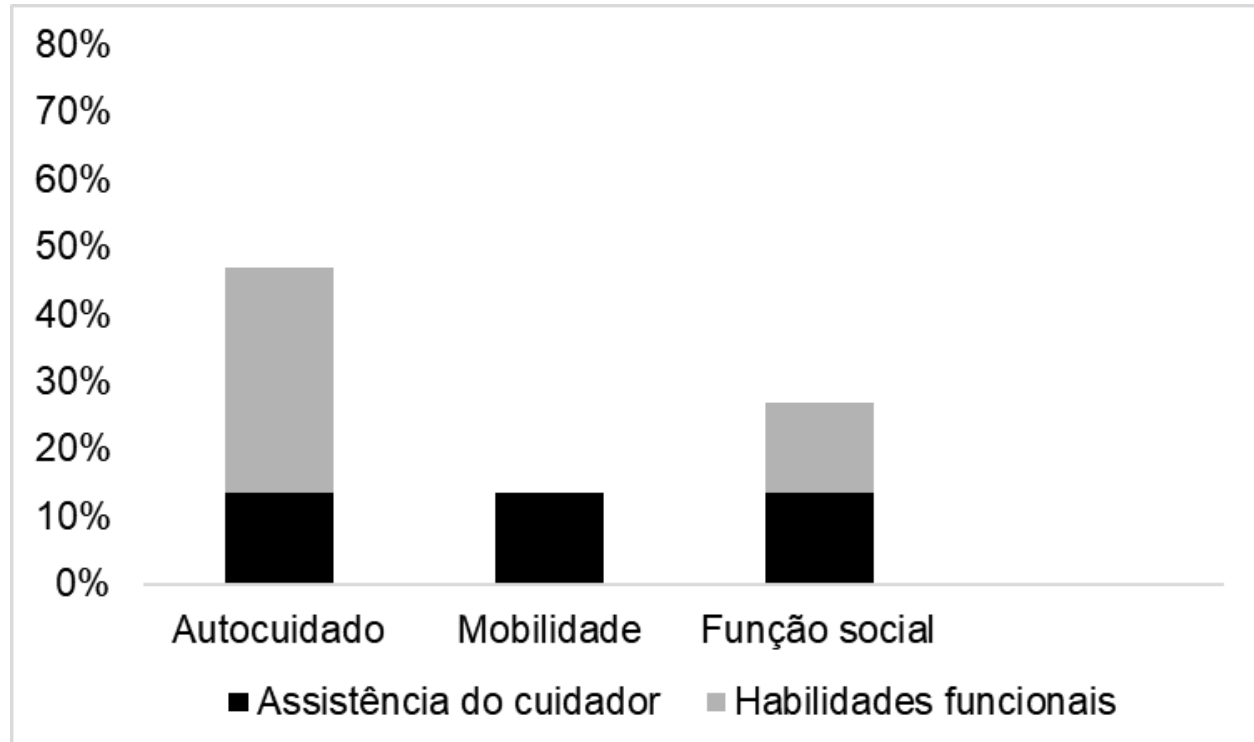

Figura 2. Descrição do desempenho nas atividades diárias e relação Habilidades Funcionais/Dependência do Cuidador nas crianças sem deficiência visual.

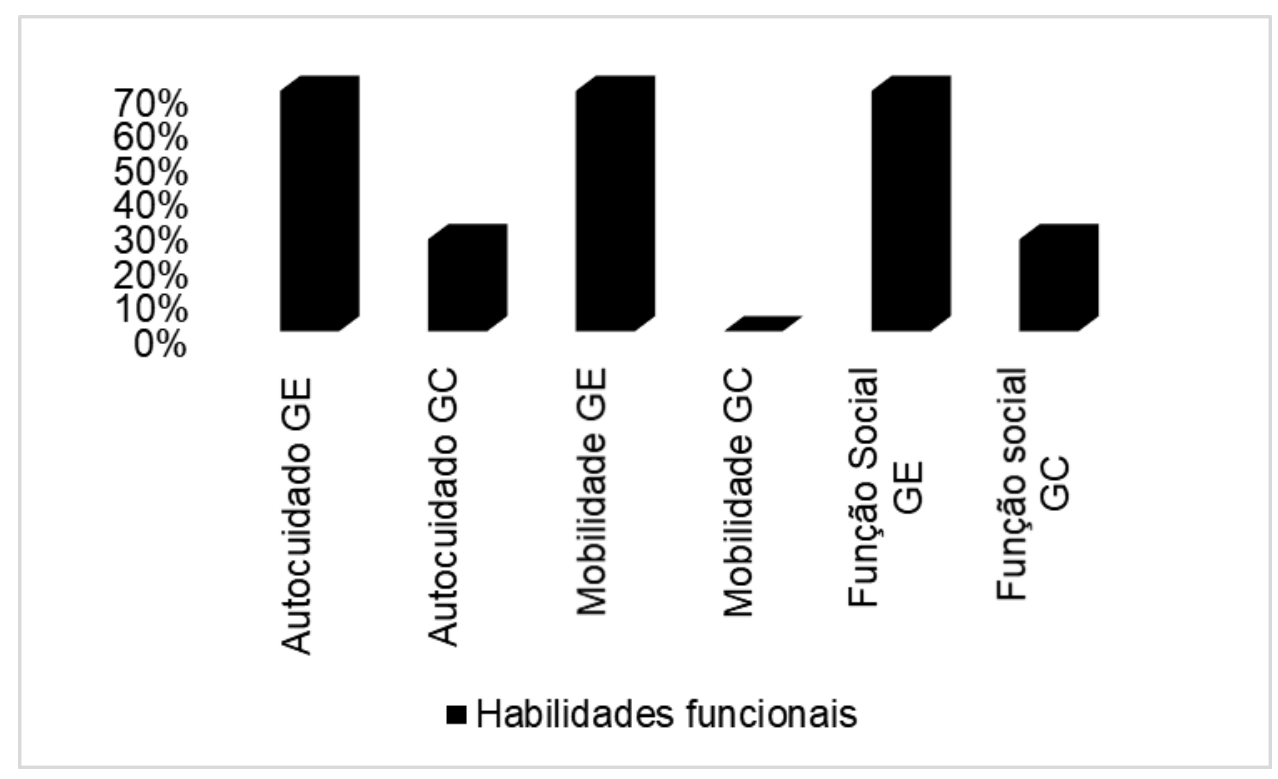

Figura 3. Descrição do desempenho das crianças do grupo de estudo e do grupo controle nas Habilidades Funcionais em Autocuidado, Mobilidade e Função social. Sendo GE-grupo de estudo e GC-grupo controle.

No desempenho das habilidades funcionais, representado na Figura 3 houve um atraso mais significativo no grupo de estudo nas três áreas Autocuidado, Mobilidade e Função social, sendo 9 crianças com atraso para desempenhar as tarefas de autocuidado (GE), 8 crianças com atraso para desempenhar as tarefas da mobilidade (GE) e 7 crianças com atraso nas tarefas da função social (GE); enquanto do grupo 
controle, 5 e 2 crianças apresentaram atraso para desempenhar as tarefas de autocuidado (GC) e da função social (GC), respectivamente. Na mobilidade, nenhuma criança teve atraso.
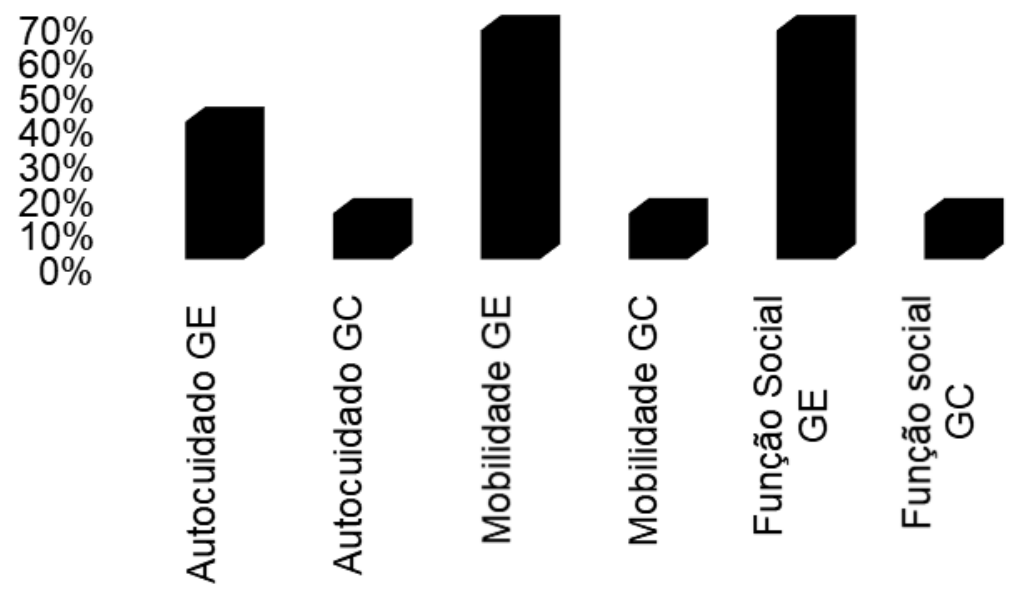

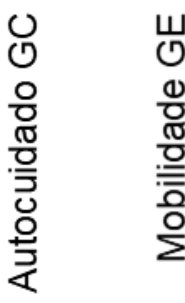

U

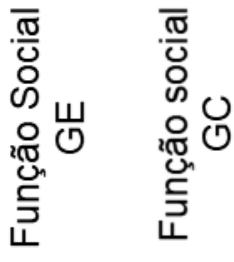

- Assistência do cuidador

Figura 4. Descrição do desempenho das crianças do grupo de estudo e do grupo controle na Assistência do Cuidador em Autocuidado, Mobilidade e Função social. Sendo GEgrupo de estudo e GC-grupo controle.

$\mathrm{Na}$ Figura 4, nota-se que as crianças do grupo de estudo, possuem maior dependência dos cuidadores na realização das atividades diárias relacionadas ao autocuidado: 6 crianças dependentes (GE) enquanto 2 crianças do grupo controle (GC); à mobilidade: 10 crianças dependentes (GE) enquanto 2 crianças do grupo controle (GC) e à função social: 10 crianças dependentes dos cuidadores (GE) e 2 crianças do grupo controle (GC).

Nas crianças do grupo controle que tiveram um atraso para desempenhar as atividades da vida diária relacionadas ao autocuidado e função social, justifica-se muitas vezes, pela jornada dupla das famílias e falta de tempo hábil para deixar a criança mais autônoma, conforme relatos dos cuidadores durante a entrevista.

As crianças com deficiência visual possuem menor desempenho para realizar suas atividades diárias, arriscam-se menos por insegurança e apresentam mais desistência para realizar as atividades relacionadas ao autocuidado, mobilidade e função social, e consequentemente, os cuidadores passam a auxiliá-las com maior frequência. Geralmente as crianças com deficiência visual, possuem essas dificuldades 
pois carregam junto à própria deficiência visual outras comorbidades que alteram sua coordenação motora, destreza, relação corpo x espaço, cognição e planejamento motor (Meereis, Lemos, Pranke, Alves, Teixeira, \& Mota, 2011; Brandão, Nascentes, \& Pereira, 2017).

A deficiência visual impõe restrições à funcionalidade e a capacidade de movimento livre, seguro e confiante da criança no ambiente. Agir autonomamente é fundamental para a criança com deficiência visual, pois o movimento é fonte de ação, da experiência, da integração sensorial, da aprendizagem e construção do conhecimento (Brasil, 2004).

Grande parte das experiências da criança na exploração do ambiente, na descoberta do corpo, na relação do corpo com os objetos e pessoas, tem participação direta da visão, que se torna um elemento fundamental na efetivação das ações da criança em seu meio ambiente (Santos, Passos, \& Rezende, 2007).

$\mathrm{Na}$ prática profissional, observa-se que a capacidade de aprendizagem por imitação está limitada pela dificuldade na elaboração de uma imagem corporal, falta de vivências corporais, falta de iniciativa, insegurança diante do desconhecido, dificuldade na integração das informações advindas dos sentidos remanescentes e da falta de oportunidades proporcionadas pela família. Assim, o desempenho das atividades básicas de vida diária fica mais difícil, e todos esses aspectos podem levar ao atraso na conquista da independência no autocuidado, no estabelecimento de rotinas, hábitos e na compreensão das mudanças (Mota, 2001).

Neste estudo, observa-se que houve alterações funcionais em ambos os grupos, e que especificamente nas crianças do grupo controle foi justificado, através de relatos dos pais, pela falta de tempo em estar junto com a criança para acompanhá-la mais em seu cotidiano, devido à dupla jornada de trabalho; e por estarem com as crianças mais no período da noite e nos finais de semana somente, sendo que, nesses momentos tentam fazer tudo pelos filhos. As alterações foram observadas principalmente na área de autocuidado relacionada às tarefas de vestir/despir, na tentativa de realizarem sozinhos como também maior dependência dos pais e/ou cuidadores para realizar estas tarefas. Estas crianças necessitam somente de incentivos e supervisão para desenvolver autonomia e conquistar independência. 
As crianças do grupo de estudo tiveram maior atraso nas atividades de autocuidado relacionadas às tarefas de alimentação (uso da colher), higienização das mãos (esfregar as mãos para lavar), vestir/despir (peças simples da parte superiorcamisetas, vestidos e inferior-calças, bermudas) e tarefas do toalete (tirar/colocar roupas, uso do vaso sanitário, limpar-se), muitos faziam uso de fraldas; seguindo para as atividades de mobilidade, relacionada às tarefas de locomoção em ambientes interno e externo. Além disso, necessitaram de mais auxílio do cuidador na área da mobilidade e na função social. Foi observado o desconhecimento da real necessidade de auxílio e das implicações da deficiência visual no desempenho para a realização das atividades diárias. O mesmo não foi observado nas crianças do grupo controle que apresentaram maior atraso e mais dependência do cuidador para desempenhar as atividades relacionadas ao autocuidado apenas, justamente pelos pais/cuidadores fazerem por eles, ser mais prático e terem mais tempo para se dedicarem às outras atividades do cotidiano, pois a maioria trabalhava; e não por uma condição patológica.

De fato, a participação da família como promotora do desempenho independente e autônomo assume um papel fundamental no processo de habilitação e reabilitação das crianças com deficiência visual (Ribeiro, 2007; Chacon, Defendi, \& Felippe, 2007) e a superproteção é vista como fator limitante para a realização das atividades diárias (Castilho, Gontijo, Alves, \& Souza, 2011).

Os dados do atual estudo não se correlacionaram com um estudo realizado em 2010 no uso do PEDI em crianças com e sem deficiência visual nas áreas de autocuidado e mobilidade relacionadas às habilidades funcionais e assistência do cuidador, na faixa etária de dois e seis anos (Mancini, Braga, Albuquerque, Ramos, \& Chagas, 2010). Neste estudo verificou que o desempenho nas habilidades funcionais de autocuidado foi diferente entre as crianças com e sem deficiência visual; no atual estudo, os dois grupos apresentaram desempenho inferior nas habilidades funcionais de autocuidado, principalmente o grupo de estudo. Na assistência do cuidador em autocuidado, observaram que as crianças com deficiência visual tiveram maior dependência para realizar as tarefas do que as crianças sem deficiência; enquanto no atual estudo, verificou-se que as crianças do grupo de estudo tiveram maior dependência do cuidador para as tarefas relacionadas à mobilidade e função social, 
enquanto as crianças do grupo controle tiveram mesmo nível de dependência do cuidador para realização das tarefas em autocuidado, mobilidade e função social.

A criança precisa ter ao seu redor condições que a estimule a se desenvolver em toda sua potencialidade. Essas condições podem ser humanas, ou seja, pessoas que promovam de forma positiva essa interação; e físicas, como moradia, parques, creches e escolas, que the permitam viver experiências diferentes e somatórias a fim de oferecer proteção, estímulo e a sensação de conquistas e realizações (Amira, Isabel, Viviana, \& Yehuda, 2005).

Conhecer os costumes, valores e condições de vida da criança deficiente visual no ambiente familiar e o grau de participação nas atividades de vida diária é fator condicionante no estabelecimento dos objetivos do programa de tratamento do terapeuta ocupacional. $\mathrm{O}$ desempenho poderá ocorrer de forma mais lenta, mas deverá ser facilitado e estimulado (Mota, 2001).

No estudo de Brandão, Nascentes e Pereira (2017), realizado em duas instituições da região do Triângulo Mineiro com crianças com e sem deficiência visual, não verificaram diferenças significativas entre os dois grupos de estudo, talvez pelo fato de as crianças com deficiência visual receberem intervenção na estimulação visual há mais de seis meses. As crianças dos dois grupos apresentaram melhor pontuação no desempenho nas habilidades funcionais do que na assistência do cuidador nas tarefas relacionadas ao autocuidado, e necessitaram de mais auxílio do cuidador nas tarefas de banheiro, questionada pelo desenvolvimento da aquisição do controle intestinal e urinário e menos auxílio nas tarefas de alimentação. No atual estudo, não se levou em consideração quanto tempo de intervenção as crianças estavam recebendo no ambulatório.

Existe uma associação entre visão e desenvolvimento, de forma que a redução das capacidades visuais pode comprometer a funcionalidade e a independência da criança, em várias fases do desenvolvimento, prejudicando algumas áreas do desenvolvimento infantil, como as relacionadas ao desempenho nas atividades de vida diária (Frutuoso, 2008).

Sendo assim, a visão, como uma de suas principais características, ajuda a integrar as diferentes modalidades sensoriais e auxiliar na compreensão das várias informações que recebemos dos sentidos (Sanchez, 1994). As atividades de vida Rev. Psicol Saúde e Debate. Jul., 2021:7(2): 113-130. 
diária, além de favorecerem a independência, ajudam na autoconfiança, autonomia e no desenvolvimento global da criança (Mota, 2001). A estimulação das atividades de vida diária deve iniciar-se o mais precocemente possível e ser desenvolvido a partir do nível de experiência perceptiva, dos significados e do nível conceptual da criança e não seguir a lógica das crianças sem deficiência visual, mas considerar as peculiaridades da cegueira e da baixa visão (Mendonça, Miguel, Neves, Micaelo, \& Reino, 2008).

\section{CONSIDERAÇÕES FINAIS}

Este estudo atingiu o objetivo principal, que foi verificar o desempenho funcional nas atividades básicas de vida diária das crianças com deficiência visual e o nível de ajuda que necessitam de seus cuidadores. Podemos levar em consideração que, as crianças com prejuízo visual apresentam maiores dificuldades e, consequentemente, menor desempenho funcional e necessitam de mais auxílio dos seus cuidadores para realizar suas atividades diárias em todas as áreas funcionais, se comparadas com crianças sem deficiência visual.

Há poucos estudos na abordagem das atividades de vida diária em crianças com deficiência visual. Acreditamos ser válido mais pesquisas voltadas para a área ou até mesmo um acompanhamento dessas crianças, por um período, nas suas demandas durante o desempenho das atividades do cotidiano.

$\mathrm{Na}$ presença das dificuldades, cabe ao profissional terapeuta ocupacional identificar o impacto desse "retrato" no repertório funcional da criança, orientar pais e cuidadores da importância de estimular o desempenho mais autônomo da criança, elaborar adaptações de utensílios, vestimentas, de ambientes como facilitadores desse processo, garantindo melhor conduta terapêutica.

\section{REFERÊNCIAS}

Amira, C.F., Isabel, C.N. de S, Viviana, G.R., Yehuda, B. (2005). Manual para vigilância do desenvolvimento infantil no contexto da AIDPI. Washington: OPS. 
AOTA. (2008). Occupational therapy practice framework: Domain and process (2nd ed.). American Journal of Occupational Therapy, 62, 625-683.

Brandão, J., Nascentes, G.A.N., Pereira, K. (2017). Assistência do cuidador nas habilidades funcionais de autocuidado em crianças com baixa visão de 2 a 3 anos de idade. Rev. Bras. Oftalmol, 76 (1). [citado em 02 de setembro de 2020].Disponível em http://www.scielo.br/scielo.

Brandão, A.O., Vasconcelos, G.C., Tibúrcio, D.J., Rossi, L.D.F.R., Andrade, G.M.Q. (2019). Avaliação da funcionalidade em crianças de 4-6 anos apresentando toxoplasmose congênita e retinocoroidite. Cad Bras Ter Ocup, 27 (1), 45-53.

Boulton, M., Haines, L., Smyth, D., Fielder, A. (2006). Health-related quality of life of children with vision impairment or blindness. Dev Med Child Neurol, 48 (8), 656-61.

Brasil. Ministério da Educação. (2004). Saberes e práticas da inclusão: dificuldades de comunicação e sinalização. Deficiência Visual. Educação Infantil, v.8, Brasília: MEC/SEESP.

Bronfenbrenner, U. (2011). Bioecologia do desenvolvimento humano: tornando os seres humanos mais humanos. Porto Alegre: Artmed.

Castilho, C.N., Gontijo, D.T., Alves, H.C., Souza, A.C.A. (2011). A gente tenta mostrar e o povo não vê: análise da participação de pessoas com cegueira congênita nos diferentes ciclos da vida. Cad Ter Ocup UFSCar, 19 (2), 189-201. Disponível em: http://www.cadernosdeterapiaocupacional.ufscar.br/index.php/cadernos/article/view /461/326.

Chacon, M.C.M.; Defendi, E.L., Felippe, M.C.G.C. (2007). A família como parceira no processo de desenvolvimento e educação do deficiente visual. In EFS Masini (Org.). A pessoa com deficiência visual: um livro para educadores. São Paulo, SP: Vetor.

Chak, M., Rahi, J.S. (2007). The health-related quality of life of children with congenital cataract: findings of the British Congenital Cataract Study. British Congenital Cataract Interest Group. Br J Ophthalmol, 91 (7), 922-6.

Costa, M.F., Oliveira, A.G.F., Bergamasco, N.H.P., Ventura, D.F. (2006). Medidas psicofísicas e eletrofisiológicas da função visual do recém-nascido: uma revisão. Psicologia USP, 17 (4), 15-33.

Daw, N. (1995). Vision development. New York: Plenm Press.

Finger, J.A. (1986). Terapia ocupacional. São Paulo: Sarvier.

Frutuoso, A. (2008, julho 02). Deficiência visual na criança. Portal Retina. Disponível em: http://www.portaldaoftalmologia.com.br/home/artigos.asp?cod=94.

Gieser, J.P. (2004). When treatiment fails: caring for patients whith visual disability. Arch Ophthalmol, 122, 1208-1209.

Graziano, R.M., Leone, C.R. (2005). Problemas oftalmológicos mais frequentes e desenvolvimento visual do pré-termo extremo. J Pediatr, 81 (1), 95-100.

Rev. Psicol Saúde e Debate. Jul., 2021:7(2): 113-130. 
Haddad, M.A.O., Correa, L.F.J., Wilson, S.M., Newton, K.J. (2006). Pediatric and adolescent population with visual impairment: study of 385 cases. Clinics, 61 (3), 239-46.

Kwon, M.Y., Legge, G.E., Fang, F., Cheong, A.M.Y., He, S. (2009). Adaptive changes in visual córtex following prolonged contrast reduction. Journal of Vision, 9 (2),1-16.

Lipsitt, L.P. (1986). Learning in infancy: cognitive development in babies. J Pediatr, 109, $172-82$.

Lopes, M.C.B. (2014). Relação entre o Questionário de Função Visual Infantil e as medidas psicofísicas de acuidade visual e visão de cores em crianças com deficiência visual. Tese de doutorado, Universidade de São Paulo-USP, SP.

Mancini, M.C. (2005). Inventário de Avaliação Pediátrica de Incapacidade-Manual da Versão Brasileira Adaptada. Belo Horizonte: UFMG.

Mancini, M.C., Braga, M.A.F., Albuquerque, K.A., Ramos, T.M.V., Chagas, P.S.C. (2010). Comparação do desempenho funcional. Rev Ter Ocup Univ São Paulo, 21 (3), 215-222.

Mayer, D.L.A.S., Beiser, A.F., Warner, E.M., Pratt, K.N., Raye, M., Lang, J.M. (1995). Monocular acuity norms for the Teller Acuity Cards between ages one month and four years. Investigative Ophthalmology \& Visual Science, 36 (3), 671-685.

Mendonça, A., Miguel, C., Neves, G., Micaelo, M., Reino, V. (2008). Alunos cegos e com Baixa Visão. Orientações curriculares. Ministério da educação.

Meereis, E.C.W., Lemos, L.F.C., Pranke, G.I., Alves, R.F., Teixeira, C.S., Mota, C.B. (2011). Deficiência visual: uma revisão focada no equilíbrio postural, desenvolvimento psicomotor e intervenções. Rev Bras Ci e Mov, 19 (1), 108-113.

Mota, M.P. (2001). Atividades da vida diária: importante instrumento na habilitação do deficiente visual. Mundo Saúde, 25 (4), 358-360.

Ottaiano, J.A.A., Ávila, M.P., Umbelino, A.C.T. (2019). As condições de saúde ocular no Brasil. São Paulo: Conselho Brasileiro de Oftalmologia.

Ribeiro, L.B. (2007). Disfunção visual. In A Cavalcanti \& C Galvão (Orgs.). Terapia ocupacional: fundamentação \& prática. Rio de Janeiro, RJ: Guanabara Koogan.

Sanchez, P.A. (1994). Deficiencias visuales y psicomotricidade: teoria y pratica. Organización Nacional de Ciegos Españoles.

Santos, L.C., Passos, J.E.O.S., Rezende, A.L.G. (2007). Os efeitos da aprendizagem psicomotora no controle das atividades de locomoção sobre obstáculos em crianças com deficiência da visão. Rev Bras Educ Espec, 13 (3), 365-380.

Salomão, S.R., Ventura, D.F. (1995). Large scale population age norms for visual acuities obtained with Vistech/Teller acuity cards. Investigative Ophthalmology Visual Science, 36 (3), 657-670. 
Scholl, G.T. (1986). Growth and development. In: Foundations of education for blind and visually handcapped children and youth: Theory and Practice. New York: American Foundation for the Blind.

Silva, M.R., Airoldi, M.J. (2014). Influência do familiar na aquisição de habilidades funcionais da criança com deficiência visual. Rev Ter Ocup USP, 25 (1), 36-42.

Vasconcelos, T.B., Cavalcante, L.I.C. (2013). Avaliação das atividades de vida diária. Rev Ter Ocup Univ São Paulo, Set-dez, 24 (3), 267-272.

Verzoni, D.S., Zin, A.A., Barbosa, A.D.M. (2017). Causes of visual impairment and blindness in children at Instituto Benjamin Constant Blind School. Rev Bras Oftalmol Rio de Janeiro, 76 (3), 138-43. 